\begin{tabular}{|l|l|l|}
\hline \multicolumn{2}{|c|}{ PublisherInfo } \\
\hline \hline PublisherName & $:$ & BioMed Central \\
\hline \hline PublisherLocation & $:$ & London \\
\hline \hline PublisherImprintName & $:$ & BioMed Central \\
\hline \hline
\end{tabular}

\title{
TRAIL inhibits autoimmune inflammation
}

\begin{tabular}{|l|l|l||}
\hline \multicolumn{2}{|c|}{ ArticleInfo } \\
\hline \hline ArticleID & $:$ & 191 \\
\hline \hline ArticleDOI & $:$ & $10.1186 /$ ar-2000-66800 \\
\hline \hline ArticleCitationID & $:$ & 66800 \\
\hline \hline ArticleSequenceNumber & $:$ & 148 \\
\hline \hline ArticleCategory & $:$ & Paper Report \\
\hline ArticleFirstPage & $:$ & 1 \\
\hline \hline ArticleLastPage & $:$ & 4 \\
\hline \hline & & RegistrationDate : 2000-4-18 \\
\hline ArticleHistory & $:$ & OnlineDate \\
\hline \hline ArticleCopyright & $:$ & Current Science Ltd2000-4-18 \\
\hline \hline ArticleGrants & $:$ & \\
\hline \hline ArticleContext & $:$ & 130753311 \\
\hline \hline
\end{tabular}


Aff1 Imperial College School of Medicine, London, UK

\section{Keywords}

Apoptosis, autoimmunity, cell cycle, death receptor, inflammation, TRAIL

\section{Context}

TRAIL is a type II membrane protein of the tumor necrosis factor (TNF) superfamily, which interacts with a variety of receptors, including DRs 4 and 5, decoy receptors (DcR1 and -2) and the soluble receptor osteoprotegerin. In vitro, TRAIL induces the apoptosis of some, but not all, tumor cell lines via DR4 and DR5. The decoy receptors, which do not contain functional death domains, block TRAILinduced apoptosis. In vivo administration of recombinant TRAIL selectively kills tumor and not host cells, an effect which is of enormous therapeutic interest. To understand the role of TRAIL in both health and disease.

\section{Significant findings}

Soluble DR5 blocked the dose-dependent TRAIL-induced apoptosis of the human Jurkat T cell line. In vivo studies demonstrated that blocking TRAIL by injection of soluble DR5 into arthritic mice increased the severity of disease compared to controls, with histochemical analysis showing severe synovitis, hyperplasia of synovial membrane, cartilage and bone erosion in most synovial joints of the feet, and increased cell proliferation. To find whether TRAIL inhibits autoimmune arthritis, intraarticular TRAIL gene transfer was carried out using a replication-defective adenovirus containing the mouse TRAIL gene. The TRAIL-containing adenovirus was injected into arthritic joints 6 days after the onset of disease and resulted in a dramatic amelioration of the disease that could be neutralised by soluble DR5. Therapeutic effects lasted approximately 10 days. Lymphocyte proliferation, cytokine production and anticollagen IgG antibodies were all elevated in DR5-treated animals compared to those in controls, indicating that chronic TRAIL blockade gives rise to enhanced cellular and humoral responses. To test the theory that the pro-inflammatory effects of DR5 are due to the blockade of TRAIL-induced apoptosis of inflammatory cells, the effect of TRAIL blockade on synovial cell apoptosis in vivo was examined. There were no differences observed in apoptotic cell numbers between DR5 treated and control mice. However, TRAIL does appear to play a role in regulating cell cycle progression: addition of soluble DR5 to anti-CD3 stimulated splenocytes increased the number of cells in the S-G2/M phase of the cell cycle. Subsequent addition of recombinant TRAIL partially inhibited 
this response. Anti-CD3 stimulation of T lymphocytes induced a marked increase in tritiated thymidine incorporation, indicating G1 to S phase progression, which was enhanced by DR 5 and inhibited by TRAIL suggesting TRAIL plays a regulatory role in inhibiting the cell cycle.

\section{Comments}

In this paper the authors have begun to elucidate the mechanism by which tumor necrosis factorrelated apoptosis-inducing ligand (TRAIL) regulates autoimmune inflammation, although further studies are now required to establish the detailed molecular mechanisms through which this effect is mediated. Interestingly, they demonstrate that administration of TRAIL by gene transfer to mice with collageninduced arthritis dramatically reduces disease severity. However, unfortunately no histological analysis was carried out on the

TRAIL treated mice. Further work might include this and extend the finding to include alternative models of inflammatory disease. TRAIL has previously been identified as a potential agent for use in cancer therapy as it selectively induces apoptosis in tumor, but not normal, cells. This study demonstrates that TRAIL may also be of therapeutic interest in the treatment of inflammatory diseases.

\section{Methods}

Recombinant soluble DR5 and a recombinant TRAIL-containing adenovirus were generated by homologous recombination. Arthritis was induced in mice through multiple injections of chicken type II collagen, and the severity of disease was subsequently scored. Paraffin embedded sections of formalin fixed joints were stained with hematoxylin and eosin and the degree of arthritic inflammation was scored. Proliferating cells within synovial joints were identified by bromodeoxyuridine (BrdU) incorporation. DNA fragments, characteristic of apoptotic cells, were detected using a digoxigenin labelling system.

\section{Additional information}

There is another interesting paper by Nicklin et al in the same edition of $J$ Exp Med describing similar mutant mice (Paper Report). 


\section{References}

1. Song K, Chen Y, Goke R, Wilmen A, Seidel C, Goke A, Hilliard B: Tumor necrosis factor-related apoptosis-inducing ligand (TRAIL) is an inhibitor of autoimmune inflammation and cell cycle progression. J Exp Med. 2000, 191: 1095-1103.

This PDF file was created after publication. 